\title{
OSCILLATORY INTEGRALS AND UNIQUE CONTINUATION FOR SECOND ORDER ELLIPTIC DIFFERENTIAL EQUATIONS
}

\author{
CHRISTOPHER D. SOGGE
}

\section{INTRODUCTION}

In this paper we establish a unique continuation theorem for solutions of equations

$$
P(x, D) u=V u
$$

with highly singular potentials $V(x)$ when $P(x, D)$ is a second order elliptic partial differential operator having bounded measurable coefficients and a real principal part with $C^{\infty}$ coefficients. With this notation our main result is the following.

Theorem 1.1. Let $n \geq 3, p=2 n /(n+2)$ and $u$ satisfy $D^{\alpha} u \in L_{\mathrm{loc}}^{p}(X), 0 \leq$ $|\alpha| \leq 2$, where $X \subset \mathbb{R}^{n}$ is a connected open set. If

$$
|P(x, D) u| \leq V|u|, \quad \text { in } X,
$$

and $V \in L_{\mathrm{loc}}^{n / 2}$, then $u$ is identically 0 if it vanishes in a nonempty open subset.

A well-known counterexample (see [11]) indicates that the condition $V \in$ $L_{\text {loc }}^{n / 2}$ is in the best possible nature. This counterexample, however, has to do with unique continuation from a point rather than from open sets as in Theorem 1.1. We hope to treat unique continuation from a point in a subsequent paper.

Results of this type for constant coefficient operators were obtained by Sawyer [14] for low dimensions and Jerison and Kenig [11] for general $n$. There was a lot of previous work, and we refer the reader to [11, 14] for further references. Also, Jerison and Kenig proved the stronger result that there is unique continuation from a point for constant coefficient operators.

For variable coefficient operators our results improve one of Hörmander [8] which says that there is unique continuation from open sets for solutions to (1.1) when $V \in L_{\text {loc }}^{(4 n-2) / 7}$ and $n \geq 5$. This result is weaker since $L_{\text {loc }}^{(4 n-2) / 7} \subset L_{\text {loc }}^{n / 2}$; however, Hörmander's theorem requires weaker regularity assumptions on the principal part of $P(x, D)$. Also, it will be clear from the proof of Theorem 1.1

Received by the editors April 19, 1988.

1980 Mathematics Subject Classification (1985 Revision). Primary 35B45, 35B60.

Supported by an NSF postdoctoral fellowship. This research was carried out in part at the I.H.E.S. and M.S.R.I., whom we would like to thank. 
that we could relax the assumptions on the lower order terms of $P(x, D)$, but, since these assumptions would evidently not be optimal, we have chosen not to state them.

We shall prove this unique continuation theorem by using the method of Carleman inequalities. Specifically, after making a natural choice of coordinates, we shall see that it suffices to show that, if $Q(x, D)$ is of the form

$$
Q(x, D)=D_{1}^{2}+\sum_{j, k \geq 2} g^{j k}(x) D_{j} D_{k}
$$

(where $D_{j}=\frac{1}{i} \frac{\partial}{\partial x_{j}}$ ) and if $w(x)=x_{1}+x_{1}^{2} / 2$, then

$$
\begin{aligned}
\left\|e^{\tau w} u\right\|_{p^{\prime}} & \leq C\left\|e^{\tau w} Q(\varepsilon x, D) u\right\|_{p}, \\
\tau^{1 / n}\left\|e^{\tau w} \nabla u\right\|_{p} & \leq C\left\|e^{\tau w} Q(\varepsilon x, D) u\right\|_{p}
\end{aligned}
$$

whenever $u$ is supported in a small enough neighborhood $X$ containing 0 and $\varepsilon$ and $1 / \tau$ are sufficienily small and positive. Here and throughout $p^{\prime}$ denotes the dual of $p$. The convexity of $w$ and positivity of $\tau$ are crucial (see Remark 2.5 ). In fact, the variant of the second inequality with $\tau^{1 / n}$ being replaced by 1 would not even hold if $w$ were linear. On account of this the techniques of Kenig et al. [12], which dealt with Carleman inequalities involving linear weights and constant coefficient operators, cannot be used here.

In the past, variable coefficient results were derived from the following $L^{2}$ Carleman inequality for the Laplace operator, $\Delta=-\sum_{j=1}^{n} D_{j}^{2}$, in $\mathbb{R}^{n}$

$$
\sum_{|\alpha| \leq 2} \tau^{3 / 2-|\alpha|}\left\|e^{\tau w} D^{\alpha} u\right\|_{2} \leq C\left\|e^{\tau w} \Delta u\right\|_{2}, \quad u \in C_{0}^{\infty}(X) .
$$

Hörmander's proof of this inequality is based on the Treves identity (see [9, Lemma 17.2.2]) and $L^{2}$ methods, both of which do not seem to be useful for proving (1.3).

Any of these inequalities would follow from showing that the conjugated operator

$$
Q_{\tau}(\varepsilon x, D)=e^{\tau w} Q(\varepsilon x, D) e^{-\tau w}
$$

satisfies the relevant local Sobolev inequalities (cf. (2.3)). One way of proving these would be to find a local parametrix for $Q_{\tau}$ which, hopefully, one could favorably estimate (along with its associated remainder) using $L^{p}$ methods. This is exactly what we shall do. Our approach is related in spirit to the proof of the Calderón uniqueness theorem (see [9, Chapter 28]); however, since we are dealing with $L^{p}$ rather than $L^{2}$, it does not seem that we can apply commutator arguments and so more attention will have to be given to parametrix constructions. After constructing the parametrix, we can make the necessary estimates by adapting arguments in Jerison's [10] simplified proof of the theorem in [11]. In addition to proving (1.3), we shall use our techniques to give a new proof of the Carleman inequality (1.4). 
Notice that $Q_{\tau}(\varepsilon x, D)=Q\left(\varepsilon x, D+i \tau w^{\prime}\right)$ and hence has a symbol

$$
Q_{\tau}(\varepsilon x, \xi)=Q\left(\varepsilon x, \xi+i \tau w^{\prime}\right)+\tau w^{\prime \prime} .
$$

(We are abusing notation a bit by letting $w^{\prime}$ denote the gradient of $w$ as well as $\partial w / \partial x_{1}$, but this will always be clear in the context.) The difficulty in constructing a parametrix which will provide good $L^{p}$ estimates as $\tau \rightarrow+\infty$ is that the "main term", $Q\left(\varepsilon x, \xi+i \tau w^{\prime}\right)$, of the symbol of $Q_{\tau}$ always vanishes on a submanifold $\Sigma_{\tau} \subset X \times \mathbb{R}^{n}$ of codimension two which is contained in the set $\left\{(x, \xi) \in X \times \mathbb{R}^{n}:|\xi| \in\left[c_{1} \tau, c_{2} \tau\right]\right\}$ for some fixed positive constants $c_{j}$. On account of this (cf. [16]), one suspects that the parametrix should involve singular Fourier integrals. This will be the case, and, in fact, we shall see that the main term involves a Fourier integral with a complex phase function whose imaginary part is bounded below by a positive multiple of $\left(x_{1}-y_{1}\right)^{2}\left|\xi^{\prime}\right|$, where $\xi^{\prime}=\left(\xi_{2}, \ldots, \xi_{n}\right)$. This last fact will turn out to be very useful for us, since, as we shall see from stationary phase, the main term is essentially the tensor product of an approximation to the identity (with kernel of "width" $\tau^{-1 / 2}$ ) acting on the first variable and an oscillatory integral operator acting on the other variables which is very similar to the ball multiplier operator in Fourier analysis (see Remark 3.8). Taking all of this into account, we shall be able to argue essentially as in Sogge $[15,16]$, using the oscillatory integral theorems arising in Bochner-Riesz summation, to obtain the desired estimates.

In order to motivate the parametrix construction, let us recall how one can use Fourier integrals to obtain a local (right) inverse for operators of the form

$$
P_{m}(x, D)-\tau^{m}
$$

where $P_{m}$ is an $m$ th order elliptic differential operator with $C^{\infty}$ coefficients and positive symbol. ${ }^{1}$ In many problems (see e.g. [16]) one wishes to have a parametrix whose error terms can be estimated in some uniform sense as $\tau \rightarrow+\infty$, and the usual parametrix constructions involving pseudodifferential operators $($ see $[9$, p. 73$])$ are inadequate. On the other hand, one can construct a parametrix whose leading term is a Fourier integral of the form

$$
(2 \pi)^{-n} \int \frac{e^{i \Phi(x, y, \xi)}}{P_{m}(y, \xi)-\tau^{m}} d \xi,
$$

for some real phase function $\Phi$ which is independent of $\tau$ and satisfies

$$
\Phi(x, y, \xi)=\langle x-y, \xi\rangle+O\left(|x-y|^{2}|\xi|\right) .^{2}
$$

Already for some problems, such as proving Sobolev inequalities which imply $L^{2}$ restriction theorems for Riemannian manifolds (see $[16,17]$ ), it suffices to consider only the leading term, and this will also turn out to be the case in our

${ }^{1}$ We are indebted to L. Hörmander for this remark.

${ }^{2}$ Note that even though this implies that the phase function $\Phi$ is equivalent to $\langle x-y, \xi\rangle$ in the usual sense (see [20]), this does not imply that the Fourier integral (1.6) is equivalent to one with phase function $\langle x-y, \xi\rangle$ since the symbol in (1.6) is singular. 
proof of (1.3). The key to these constructions is to make a proper choice of the phase function $\Phi$. To describe the one in (1.6), we let $B(x, \xi)=\sqrt[m]{P_{m}(x, \xi)}$ and notice that we can factor the symbol of the operator in (1.5) as follows

$$
P_{m}(x, \xi)-\tau^{m}=(B(x, \xi)-\tau) \cdot\left(B^{m-1}+B^{m-2} \tau+\cdots+\tau^{m-1}\right) .
$$

The second factor is uniformly elliptic in the sense that it is bounded below by a multiple of $\left(|\xi|^{m-1}+\tau^{m-1}\right)$, while the first factor vanishes for certain $|\xi| \approx \tau$. Thus it is not surprising that one uses the first factor to construct $\Phi$. In fact, for $|\xi|$ larger than some fixed constant, one chooses $\Phi$ so that it solves the eikonal equation

$$
B\left(x, \Phi_{x}(x, y, \xi)\right)=B(y, \xi),
$$

where $\Phi_{x}$ denotes the gradient with respect to the $x$ variable. Since the equation is real, standard existence theorems for first order partial differential equations imply that there is a solution, when $|x-y|$ is sufficiently small, with the properties described above.

Similar ideas based on a factorization will be used to construct a parametrix for $Q_{\tau}(\varepsilon x, D)$ whose main term will be of the form

$$
(2 \pi)^{-n} \int \beta(\xi) \frac{e^{i \Phi(x, y, \xi)}}{Q\left(\varepsilon y, \xi+i \tau w^{\prime}(y)\right)} d \xi,
$$

for appropriate cutoff functions $\beta=\beta_{\tau}$ which equal one when $Q\left(\varepsilon x, \xi+i \tau w^{\prime}\right)=0$. To describe the construction of $\Phi$ here, recall that we are assuming that $Q(x, D)$ is diagonal with respect to the first variable and hence (after possibly multiplying by -1 ) has symbol

$$
Q(x, \xi)=\xi_{1}^{2}+\sum_{j, k \geq 2} g^{j k}(x) \xi_{j} \xi_{k} .
$$

Thus, if we let

$$
a\left(\varepsilon x, \xi^{\prime}\right)=\sqrt{\sum_{j, k \geq 2} g^{j k}(\varepsilon x) \xi_{j} \xi_{k}}
$$

we see that

$$
Q\left(\varepsilon x, \xi+i \tau w^{\prime}\right)=\left(\xi_{1}-i\left(a\left(\varepsilon x, \xi^{\prime}\right)-\tau w^{\prime}(x)\right)\right) \cdot\left(\xi_{1}+i\left(a\left(\varepsilon x, \xi^{\prime}\right)+\tau w^{\prime}(x)\right)\right) .
$$

The second factor has modulus which is bounded below by a multiple of $(|\xi|+\tau)$, and so, as above, one is led to choose $\Phi$ based on the first factor. Since we shall attempt to find a right inverse for the adjoint of $Q_{\tau}$, ideally one would like $\Phi$ to solve the complex eikonal equation

$$
\frac{1}{w^{\prime}(x)}\left(\frac{\partial \Phi}{\partial x_{1}}(x, y, \xi)+i a\left(\varepsilon x, \Phi_{x^{\prime}}(x, y, \xi)\right)\right)=\frac{1}{w^{\prime}(y)}\left(\xi_{1}+i a\left(\varepsilon y, \xi^{\prime}\right)\right) \text {. }
$$

However, since this is a complex nonlinear equation with $C^{\infty}$ (rather than analytic) coefficients, in general, a solution does not exist. Nonetheless, one 
can use the almost analytic machinery developed by Treves (see $[20,21])$ to construct an approximate solution which will turn out to be sufficient for our purposes. The approximate solution $\Phi$ will have positive imaginary part, and, as before, will be of the form $\langle x-y, \xi\rangle+O\left(|x-y|^{2}|\xi|\right)$.

The plan of the paper is as follows. In $\S 2$ we shall give a new proof of (1.4) which will serve as a model for the proof of (1.3) which will be given in $\S 3$. In $\S 3$ we shall also show how Theorem 1.1 follows from our $L^{p}$ Carleman inequalities. Also, in what follows, we shall use the convention that $C$ denotes a constant which is not necessarily the same at each occurrence.

We would like to thank Hart Smith and E.M. Stein for comments which helped the exposition. The inspiration for this paper grew out of joint work with C.E. Kenig and A. Ruiz [12]. Finally we would like to thank the referee for some valuable suggestions and criticisms.

\section{Model case: $L^{2}$ Carleman inequalities}

The purpose of this section is to give a new proof of the sharp classical $L^{2}$ Carleman inequality that will serve as a model for the $L^{p}$ inequalities of $\S 3$ which will be used to establish Theorem 1.1. If we let

$$
X=\left\{x \in \mathbb{R}^{n}:|x|<1 / 4\right\}
$$

and

$$
w(x)=x_{1}+x_{1}^{2} / 2
$$

then the result is the following.

Theorem 2.1. If $u \in C_{0}^{\infty}(X)$ and $\tau>0$ is large enough, then

$$
\sum_{|\alpha| \leq 2} \tau^{3 / 2-|\alpha|}\left\|e^{\tau w} D^{\alpha} u\right\|_{L^{2}(X)} \leq C\left\|e^{\tau w} \Delta u\right\|_{L^{2}(X)} .
$$

Remark 2.2. The convexity of $w$ (and the fact that $\tau>0$ ) is crucial here. If $w$ were just a nonzero linear function then one could prove only the analogue of (2.2) where $\tau^{3 / 2}$ is replaced by $\tau^{1}$ (see [16]). Further, the bounds in (2.2) are strong enough to prove $L^{2}$ Carleman inequalities for second order elliptic differential operators whose coefficients are assumed to be only Lipschitz continuous of order one. (See Hörmander [8, p. 38].) This is the case since one can apply a localization argument which involves "freezing" the coefficients of the differential operators on balls of radius $O\left(\tau^{-1 / 2}\right)$ and then absorbing the error terms arising from the construction using (2.2). This argument would also apply to $L^{p}$ Carleman inequalities if one could prove the variant of $(2.2)$ where the $L^{2}$ norms are replaced by $L^{p}$ estimates; however, since our proof of (2.2) shows that this inequality is related to the fact that the ball multiplier operator in Fourier analysis is bounded on $L^{2}$, and since C. Fefferman [4] showed that this operator is not bounded on any other $L^{p}$ space, it seems very unlikely that the analogue of (2.2) would hold for any other $L^{p}$ spaces with the same powers 
of $\tau$. Also, it is known that Carleman inequalities of the form

$$
\left\|e^{\tau w} u\right\|_{q} \leq C\left\|e^{\tau w} \Delta u\right\|_{2}
$$

can only hold when $1 / 2-1 / q \leq 7 /(4 n-2)$ (cf. [8, 10]), which is only strong enough to prove uniqueness results involving potentials $V \in L_{\text {loc }}^{(4 n-2) / 7}$. Thus, a different approach seems to be needed for proving optimal Carleman inequalities and uniqueness theorems, and the new proof of (2.2) which we shall now give will serve as a model for this approach.

To prove (2.2) we first notice that if we set $v=e^{\tau w} u$ and

$$
Q_{\tau}(x, D)=-e^{\tau w} \Delta e^{-\tau w},
$$

then (2.2) would be a consequence of the following uniform inequality:

$$
\sum_{|\alpha| \leq 2} \tau^{3 / 2-|\alpha|}\left\|D^{\alpha} v\right\|_{2} \leq C\left\|Q_{\tau}(x, D) v\right\|_{2}, \quad v \in C_{0}^{\infty}(X) .
$$

To prove this notice that

$$
Q_{\tau}(x, D)=\sum_{j=1}^{n} D_{j}^{2}+2 \tau w^{\prime} \frac{\partial}{\partial x_{1}}-\left(\tau w^{\prime}\right)^{2}+\tau w^{\prime \prime} .
$$

Therefore, since $w^{\prime} \approx 1$ on $X$, it follows that if we let $A_{\tau}(x, D)$ be the differential operator whose adjoint equals

$$
A_{\tau}^{*}(x, D)=\left(\frac{1}{w^{\prime}(x)}\right)^{2} \sum_{j=1}^{n} D_{j}^{2}-2 \tau \frac{1}{w^{\prime}(x)} \frac{\partial}{\partial x_{1}}-\tau^{2},
$$

then it suffices to prove the following variant of (2.3):

$$
\sum_{|\alpha| \leq 2} \tau^{3 / 2-|\alpha|}\left\|D^{\alpha} v\right\|_{2} \leq C\left\|A_{\tau}(x, D) v\right\|_{2}, \quad v \in C_{0}^{\infty}(X) .
$$

We have only made this reduction to simplify the notation later on.

To prove the desired inequality $(2.5)$ we shall show that $A_{\tau}^{*}(x, D)$ has a right parametrix, $T$, with the following properties.

Lemma 2.3. For $\tau>0$ there are functions $K=K_{\tau}$ and $R=R_{\tau}$ so that

$$
A_{\tau}^{*}(x, D) K(x, y)=\delta_{0}(x-y)+R(x, y), \quad x, y \in X,
$$

where $\delta_{0}$ denotes the Dirac distribution. Furthermore, if we let $T=T_{\tau}$ be the operator with kernel $K(x, y)$, i.e.,

$$
T f(x)=\int_{X} K(x, y) f(y) d y, \quad f \in C_{0}^{\infty}(X),
$$


and $R$ be the operator with kernel $R(x, y)$, then for large $\tau>0$, the adjoints of these operators satisfy the following uniform estimates whenever $f \in C_{0}^{\infty}(X)$ :

$$
\begin{gathered}
\sum_{|\alpha| \leq 2} \tau^{3 / 2-|\alpha|}\left\|D^{\alpha} T^{*} f\right\|_{L^{2}(X)} \leq C\|f\|_{L^{2}(X)}, \\
\tau^{1 / 2}\left\|R^{*} f\right\|_{L^{2}(X)} \leq C\|f\|_{L^{2}(X)}, \\
\tau^{-1 / 2}\left\|D^{\beta} R^{*} f\right\|_{L^{2}(X)} \leq C \sum_{|\alpha| \leq|\beta|-1}\left\|D^{\alpha} f\right\|_{L^{2}}, \quad 1 \leq|\beta| \leq 2 .
\end{gathered}
$$

Before constructing the right parametrix $T$ and proving the associated estimates, let us see why Lemma 2.3 implies (2.5) (and hence Theorem 2.1). First, if as in (2.5) $v \in C_{0}^{\infty}(X)$, it follows from (2.6) that

$$
v(x)=T^{*}\left(A_{\tau} v\right)(x)-R^{*} v(x) .
$$

Also, since $A_{\tau}(x, D) v$ is also supported in $X,(2.7)$ and (2.8) imply that

$$
\begin{aligned}
\tau^{3 / 2}\|v\|_{2} & \leq \tau^{3 / 2}\left\|T^{*}\left(A_{\tau} v\right)\right\|_{2}+\tau^{3 / 2}\left\|R^{*} v\right\|_{2} \\
& \leq C\left\|A_{\tau}(x, D) v\right\|_{2}+C \tau\|v\|_{2},
\end{aligned}
$$

which of course implies (2.5) for the special case where $\alpha=0$ when $\tau$ is large enough. The other cases follow from a similar argument, except that this time one must use (2.9).

We shall construct $T$ microlocally, writing it as

$$
T=T_{0}+T_{1}
$$

where $T_{0}$ will be a pseudodifferential operator of order -2 and $T_{1}$ will be a Fourier integral operator with complex phase and a singular symbol. Also, we will find that the remainder $R$ will be the sum of two terms, and, moreover, $R f$ will essentially behave like $\nabla(T f)$.

The operator $T_{0}$ will be the microlocal (right) inverse of $A_{\tau}^{*}(x, D)$ corresponding to $(x, \xi) \in X \times \mathbb{R}^{n}$ with $\xi$ being sufficiently far from the zero set of the symbol $A_{\tau}^{*}(x, \xi)$. To construct $T_{0}$ we shall use the calculus of pseudodifferential operators, and so we recall the usual condition on symbols.

Definition 2.4. We denote by $S^{m}$ the set of all $p \in C^{\infty}\left(X \times X \times \mathbb{R}^{n}\right)$ satisfying

$$
\left|D_{x, y}^{\beta} D_{\xi}^{\alpha} p(x, y, \xi)\right| \leq C_{\alpha, \beta}(1+|\xi|)^{m-|\alpha|}, \quad x, y \in X, \xi \in \mathbb{R}^{n} .
$$

Also a subset $B$ of $S^{m}$ will be said to be bounded if the same constants can be used in (2.12) for all $p \in B$.

Given a symbol $p \in S^{m}$ we associate the pseudodifferential operator

$$
(p(x, D) f)(x)=(2 \pi)^{-n} \int_{X} \int_{\mathbb{R}^{n}} p(x, y, \xi) e^{i\langle x-y, \xi\rangle} f(y) d \xi d y .
$$

With an abuse of notation we shall say that the operator $p(x, D)$ (or the kernel of the operator) is in $S^{m}$ if the symbol $p(x, y, \xi)$ belongs to $S^{m}$. 
We now turn to the proof of Lemma 2.3. The first step is to notice that the operator $A_{\tau}^{*}$ defined in (2.4) has a symbol which can be factored as follows:

$$
A_{\tau}^{*}(x, \xi)=B_{\tau}(x, \xi) \cdot G_{\tau}(x, \xi),
$$

where

$$
B_{\tau}(x, \xi)=\frac{1}{w^{\prime}(x)} \xi_{1}+i\left(\frac{1}{w^{\prime}(x)}\left|\xi^{\prime}\right|-\tau\right)
$$

and

$$
G_{\tau}(x, \xi)=\frac{1}{w^{\prime}(x)} \xi_{1}-i\left(\frac{1}{w^{\prime}(x)}\left|\xi^{\prime}\right|+\tau\right) .
$$

The "good" factor $G_{\tau}(x, \xi)$ never vanishes, and satisfies $\left|G_{\tau}(x, \xi)\right| \geq$ $\frac{1}{2}(|\xi|+\tau),(x, \xi) \in X \times \mathbb{R}^{n}$. The "bad" factor $B_{\tau}(x, \xi)$ cannot be bounded from below and in fact vanishes on the set

$$
\Sigma_{\tau}=\left\{(x, \xi) \in X \times \mathbb{R}^{n}: \xi_{1}=0,\left|\xi^{\prime}\right|=w^{\prime}(x) \tau\right\} .
$$

Nonetheless, $B_{\tau}(x, \xi)$, and hence $A_{\tau}^{*}(x, \xi)$, can be bounded from below when $(x, \xi)$ is sufficiently far from $\Sigma_{\tau}$. For instance, if we define

$$
\Gamma_{\tau}=\left\{(x, \xi) \in X \times \mathbb{R}^{n}:\left|\xi^{\prime}\right| \in[\tau / 4,4 \tau],\left|\xi_{1}\right| \leq \tau / 4\right\},
$$

and recall (2.1), then it follows that there must be a positive constant $c$ which is independent of $\tau$, so that

$$
\left|A_{\tau}^{*}(x, \xi)\right| \geq c\left(|\xi|^{2}+\tau^{2}\right), \quad \text { when }(x, \xi) \in{ }^{c} \Gamma_{\tau},
$$

where ${ }^{c} \Gamma_{\tau}$ denotes the complement of $\Gamma_{\tau}$.

We shall use (2.18) to microlocally invert $A_{\tau}^{*}(x, D)$ away from $\Gamma_{\tau}$. To this end let $\beta \in C_{0}^{\infty}(\mathbb{R})$ satisfy $\beta(s)=1$ if $|s| \in[1 / 4,4]$ and $\beta=0$ near the origin. We then define $\beta_{0}=\beta_{0, \tau}$ by

$$
\beta_{0}(\xi)=1-\beta\left(\left|\xi^{\prime}\right| / \tau\right) \beta\left(1-\xi_{1} / \tau\right)
$$

and notice that $\beta_{0}(\xi)=0$ on $\Gamma_{\tau}$. Consequently, if we define

$$
K_{0}(x, y)=(2 \pi)^{-n} \int \beta_{0}(\xi) \frac{e^{i\langle x-y, \xi\rangle}}{A_{\tau}^{*}(y, \xi)} d \xi
$$

and recall (2.18), then it follows from standard microlocal arguments (see e.g., $[9$, Chapter 18]) that

$$
A_{\tau}^{*}(x, D) K_{0}(x, y)=(2 \pi)^{-n} \int \beta_{0}(\xi) e^{i\langle x-y, \xi\rangle} d \xi+R_{0}(x, y),
$$

where $R_{0}$ belongs to a bounded subset of $S^{-1}$ (which is independent of $\tau$ ). Thus, since the adjoint operator $R_{0}^{*}$ also has this property, it follows that whenever $f \in C_{0}^{\infty}(X)$

$$
\left\|D^{\beta} R_{0}^{*} f\right\|_{L^{2}(X)} \leq C \sum_{|\alpha| \leq|\beta|-1}\left\|D^{\alpha} f\right\|_{L^{2}(X)}, \quad 1 \leq|\beta| \leq 2 .
$$


Also, since it is easy to check that $\tau R_{0}$ belongs to a bounded subset of $S^{0}$, we have that

$$
\tau\left\|R_{0}^{*} f\right\|_{L^{2}(X)} \leq C\|f\|_{L^{2}(X)},
$$

while (2.18) and (2.20) imply that, if we let $T_{0}$ be the operator with kernel $K_{0}(x, y)$, then

$$
\sum_{|\alpha| \leq 2} \tau^{2-|\alpha|}\left\|D^{\alpha} T_{0}^{*} f\right\|_{L^{2}(X)} \leq C\|f\|_{L^{2}(X)} .
$$

Inverting $A_{\tau}^{*}(x, D)$ on the set $\Gamma_{\tau}$ is much harder and will require the use of Fourier integrals with complex phase. It is for this step that we shall have to use the factorization (2.13). If $\beta_{1}=\beta_{1, \tau}$ is given by

$$
\beta_{1}(\xi)=1-\beta_{0}(\xi)
$$

where $\beta_{0}$ is as in (2.19), we would like to construct a Fourier integral operator $T_{1}$ with kernel

$$
K_{1}(x, y)=(2 \pi)^{-n} \int \beta_{1}(\xi) \frac{e^{i \Phi(x, y, \xi)}}{A_{\tau}^{*}(y, \xi)} d \xi
$$

so that the analogues of (2.21) and (2.7)-(2.9) are satisfied. Since the factor $G_{\tau}(x, \xi)$ is bounded from below on $\Gamma_{\tau}$, as we discussed in the introduction, we should expect to construct the phase function $\Phi$ in (2.22) using the other factor, $B_{\tau}(x, \xi)$. Specifically, we would like $\Phi$ to satisfy the equation

$$
B_{\tau}\left(x, \Phi_{x}\right)=B_{\tau}(y, \xi), \quad y \in X,(x, \xi) \in \Gamma_{\tau},
$$

which leads to the complex eikonal equation

$$
\frac{1}{w^{\prime}(x)} \frac{\partial \Phi}{\partial x_{1}}(x, y, \xi)+\frac{i}{w^{\prime}(x)}\left|\Phi_{x^{\prime}}(x, y, \xi)\right|=\frac{1}{w^{\prime}(y)} \xi_{1}+\frac{i}{w^{\prime}(y)}\left|\xi^{\prime}\right| \text {. }
$$

However, since $w^{\prime}(x)=1+x_{1}$, one sees that the phase function

$$
\Phi(x, y, \xi)=\langle x-y, \xi\rangle+\frac{1}{2\left(1+y_{1}\right)}\left(x_{1}-y_{1}\right)^{2} \xi_{1}+i \frac{1}{2\left(1+y_{1}\right)}\left(x_{1}-y_{1}\right)^{2}\left|\xi^{\prime}\right|
$$

solves these equations.

To use this, we note that $(2.4)$ implies that

$$
A_{\tau}^{*}(x, D) e^{i \Phi(x, y, \xi)}=e^{i \Phi} A_{\tau}^{*}\left(x, \Phi_{x}\right)+e^{i \Phi}\left(\frac{1}{w^{\prime}(x)}\right)^{2} \frac{\partial^{2} \Phi}{\partial x_{1}^{2}}
$$

Next, if we set

$$
\begin{aligned}
r(x, y, \xi) & =G_{\tau}(y, \xi)-G_{\tau}(x, \xi) \\
& =\left(\frac{1}{w^{\prime}(y)} \xi_{1}-i \frac{1}{w^{\prime}(y)}\left|\xi^{\prime}\right|\right)-\left(\frac{1}{w^{\prime}(x)} \xi_{1}-i \frac{1}{w^{\prime}(x)}\left|\xi^{\prime}\right|\right)
\end{aligned}
$$

then it follows from (2.13) and (2.23) that

$$
A_{\tau}^{*}\left(x, \Phi_{x}\right)=A_{\tau}^{*}(y, \xi)+B_{\tau}(y, \xi) r(x, y, \xi),
$$


and consequently (2.25)-(2.27) imply that if $K_{1}$ is as in (2.22)

$$
\begin{aligned}
(2 \pi)^{n} & A_{\tau}^{*}(x, D) K_{1}(x, y) \\
= & \int \beta_{1}(\xi) e^{i \Phi} d \xi+\int \beta_{1}(\xi) \frac{r(x, y, \xi)}{G_{\tau}(y, \xi)} e^{i \Phi} d \xi \\
& +\frac{1}{\left(w^{\prime}(x)\right)^{2}} \int \beta_{1}(\xi) \frac{\partial^{2} \Phi / \partial x_{1}^{2}}{A_{\tau}^{*}(y, \xi)} e^{i \Phi} d \xi .
\end{aligned}
$$

Next, notice that the phase function $\Phi$ satisfies $\Phi(x, y, \xi)=\langle x-y, \xi\rangle+$ $O\left(|x-y|^{2}|\xi|\right)$ and, hence,

$$
\nabla_{\xi} \Phi(x, y, \xi) \neq 0 \quad \text { unless } x=y \text {. }
$$

Thus, it follows from the theory of Fourier integrals (see [6, pp. 201-202; 20, $\S 17.6])$ that

$$
\int \beta_{1}(\xi) e^{i \Phi(x, y, \xi)} d \xi-\int \beta_{1}(\xi) e^{i\langle x-y, \xi\rangle} d \xi
$$

belongs to a bounded subset of $S^{-1}$, and, furthermore, on account of the support properties of $\beta_{1}(\xi), \tau$ times this difference belongs to a bounded subset of $S^{0}$. Similarly, since $r(x, y, \xi) \in S^{1}$ and

$$
r(x, y, \xi)=0 \quad \text { when } x=y,
$$

it also follows from (2.29) that the second summand in (2.28) also has these properties. To summarize, we have just seen that $(2.28)$ can be rewritten in the more favorable way

$$
A_{\tau}^{*}(x, D) K_{1}(x, y)=(2 \pi)^{-n} \int \beta_{1}(\xi) e^{i\langle x-y, \xi\rangle} d \xi+R_{1,0}(x, y)+R_{1,1}(x, y),
$$

where

$$
R_{1,1}(x, y)=\frac{(2 \pi)^{-n}}{\left(w^{\prime}(x)\right)^{2}} \int \beta_{1}(\xi) \frac{\partial^{2} \Phi / \partial x_{1}^{2}}{A_{\tau}^{*}(y, \xi)} e^{i \Phi} d \xi,
$$

while $R_{1,0}$ belongs to a bounded subset of $S^{-1}$ and $\tau R_{1,0}$ belongs to a bounded subset of $S^{0}$.

On account of this formula, we see that if we let $K(x, y)=K_{0}(x, y)+$ $K_{1}(x, y)$ and $R(x, y)=R_{0}(x, y)+R_{1}(x, y)$, where $R_{1}=R_{1,0}+R_{1,1}$, then (2.6) must hold. Thus, since $R_{1,0}$ must satisfy the analogue of (2.7.0)-(2.9.0), we see from Minkowski's inequality that, in order to finish the proof of Lemma 2.3, it suffices to show that for $f \in C_{0}^{\infty}(X)$ one has

$$
\begin{gathered}
\sum_{|\alpha| \leq 2} \tau^{3 / 2-|\alpha|}\left\|D^{\alpha} T_{1}^{*} f\right\|_{L^{2}(X)} \leq C\|f\|_{L^{2}(X)}, \\
(2.8 .1) \quad \tau^{1 / 2}\left\|R_{1,1}^{*} f\right\|_{L^{2}(X)} \leq C\|f\|_{L^{2}(X)}, \\
(2.9 .1) \quad \tau^{-1 / 2}\left\|D^{\beta} R_{1,1}^{*} f\right\|_{L^{2}(X)} \leq C \sum_{|\alpha| \leq|\beta|-1}\left\|D^{\alpha} f\right\|_{L^{2}(X)}, \quad 1 \leq|\beta| \leq 2 .
\end{gathered}
$$


However, since $R_{1,1} \approx \tau T_{1}$, we shall only demonstrate the special case

$$
\tau^{3 / 2}\left\|T_{1}^{*} f\right\|_{L^{2}(X)} \leq C\|f\|_{L^{2}(X)} .
$$

To make this estimate we first note that Minkowski's integral inequality and (2.1) imply that

$$
\left\|T_{1}^{*} f\right\|_{L^{2}\left(\mathbb{R}^{n-1}, d x^{\prime}\right)} \leq \int_{-1 / 4}^{1 / 4}\left\|\int_{\mathbb{R}^{n-1}} K_{1}^{*}(x, y) f(y) d y^{\prime}\right\|_{L^{2}\left(\mathbb{R}^{n-1}\right)} d y_{1},
$$

where $K_{1}^{*}(x, y)=\overline{K_{1}(y, x)}$. To estimate the inner integral, we recall that (2.22) and (2.24) imply that

$$
K_{1}^{*}(x, y)=(2 \pi)^{-n} \int_{\mathbb{R}^{n-1}} e^{i\left\langle x^{\prime}-y^{\prime}, \xi^{\prime}\right\rangle} m\left(x_{1}, y_{1}, \xi^{\prime}\right) d \xi^{\prime},
$$

where

$$
m\left(x_{1}, y_{1}, \xi^{\prime}\right)=\int_{-\infty}^{\infty} \beta_{1}(\xi) \frac{e^{i\left[\left(x_{1}-y_{1}\right) \xi_{1}+\left(x_{1}-y_{1}\right)^{2}\left(i\left|\xi^{\prime}\right|-\xi_{1}\right) / 2\left(1+x_{1}\right)\right]}}{|\xi|^{2} /\left(1+x_{1}\right)^{2}+2 i \tau \xi_{1} /\left(1+x_{1}\right)-\tau^{2}} d \xi_{1} .
$$
fixed

Consequently, it follows from Plancherel's theorem that when $x_{1}$ and $y_{1}$ are (2.33)

$$
\left\|\int_{\mathbb{R}^{n-1}} K_{1}^{*}(x, y) f(y) d y^{\prime}\right\|_{L^{2}\left(\mathbb{R}^{n-1}\right)} \leq \sup _{\xi^{\prime}}\left|m\left(x_{1}, y_{1}, \xi^{\prime}\right)\right| \cdot\left(\int_{\mathbb{R}^{n-1}}|f(y)|^{2} d y^{\prime}\right)^{1 / 2} .
$$

To use this, we first notice that for every $N$

$$
e^{-\left(x_{1}-y_{1}\right)^{2}\left|\xi^{\prime}\right| / 2\left(1+x_{1}\right)} \leq C_{N}\left(1+\tau\left(x_{1}-y_{1}\right)^{2}\right)^{-N} \text { on } \operatorname{supp} \beta_{1},
$$

and so, since it is easy to check that

$$
\int_{-\infty}^{\infty} \beta_{1}(\xi) \frac{e^{i \xi_{1}\left[\left(x_{1}-y_{1}\right)-\left(x_{1}-y_{1}\right)^{2} / 2\left(1+x_{1}\right)\right]}}{|\xi|^{2} /\left(1+x_{1}\right)^{2}+2 i \tau \xi_{1} /\left(1+x_{1}\right)-\tau^{2}} d \xi_{1}=O\left(\tau^{-1}\right),
$$

we find that

$$
\left|m\left(x_{1}, y_{1}, \xi^{\prime}\right)\right| \leq C \tau^{-1}\left(1+\tau\left(x_{1}-y_{1}\right)^{2}\right)^{-1} .
$$

Thus, (2.33) implies that

$$
\begin{aligned}
& \left\|T_{1}^{*} f\right\|_{L^{2}(X)} \\
& \quad \leq C \tau^{-1}\left(\int\left|\int\left(1+\tau\left(x_{1}-y_{1}\right)^{2}\right)\left\|f\left(y_{1}, \cdot\right)\right\|_{L^{2}\left(\mathbb{R}^{n-1}\right)} d y_{1}\right|^{2} d x_{1}\right)^{1 / 2} \\
& \quad \leq C \tau^{-3 / 2}\|f\|_{L^{2}\left(\mathbb{R}^{n}\right)}
\end{aligned}
$$

as desired. The last inequality follows from Young's inequality and the fact that

$$
\int_{-\infty}^{\infty}\left(1+\tau x_{1}^{2}\right)^{-1} d x_{1} \leq C \tau^{-1 / 2} .
$$

This completes the proof. 
Remark 2.5. Let us say a few words about why it is crucial to take $\tau>0$ in our Carleman inequalities. This is because even though the symbol of the operator $A_{\tau}(x, D)$ in (2.5) vanishes on the set $\Sigma_{\tau}$ in (2.16), it nonetheless satisfies the condition for subellipticity:

$$
\frac{1}{i}\left\{A_{\tau}, \bar{A}_{\tau}\right\}<0 \quad \text { on } \Sigma_{\tau},
$$

where $\{\cdot, \cdot\}$ denotes the Poisson brackets. Thus, (2.2) and (2.5) can be thought of as a subelliptic estimate with $1 / 2$ loss of derivatives which is uniform in $\tau$. In a similar vein, the reader may have noticed the similarity between our parametrix constructions and the construction of the right inverse for the Mizohata operator $D_{t}-i t\left|D_{x}\right|$ (see [20, pp. 596-602]).

\section{3. $L^{p}$ CARleman INeQualities AND UNIQUe CONTINUATION}

We shall begin with the $L^{p}$ analogue of Theorem 2.1 that will be used to prove Theorem 1.1.

Theorem 3.1. Let $n \geq 3, p=2 n /(n+2)$ and $p^{\prime}=2 n /(n-2)$ (so that $1 / p-$ $\left.1 / p^{\prime}=2 / n\right)$. Fix a second order elliptic differential operator on $\mathbb{R}^{n}$ of the form

$$
Q(x, D)=D_{1}^{2}+\sum_{j, k \geq 2} g^{j k}(x) D_{j} D_{k},
$$

where the $g^{j k}$ are real and $C^{\infty}$ in a neighborhood of 0 . Then if $X=\left\{x \in \mathbb{R}^{n}\right.$ : $|x|<\varepsilon\}$ and $w(x)=x_{1}+x_{1}^{2} / 2$, whenever $u \in C_{0}^{\infty}(X)$ and $0<\varepsilon, 1 / \tau<1 / 2$, the following inequalities are smaller than a fixed constant:

$$
\begin{gathered}
\left\|e^{\tau w} u\right\|_{L^{p^{\prime}}(X)} \leq C\left\|e^{\tau w} Q(\varepsilon x, D) u\right\|_{L^{p}(X)}, \\
\sum_{|\alpha| \leq 1} \tau^{(1+1 / n)-|\alpha|}\left\|e^{\tau w} D^{\alpha} u\right\|_{L^{p}(X)} \leq C\left\|e^{\tau w} Q(\varepsilon x, D) u\right\|_{L^{p}(X)}
\end{gathered}
$$

Remark 3.2. Homogeneity considerations show that (3.2) can never hold when $1 / p-1 / p^{\prime}>2 / n$ and that the left-hand side of (3.2) can never be dominated by $o(1)$ times the right-hand side. Also, it seems likely that the bounds in (3.3) cannot be improved. Nonetheless, (3.3) implies that the conjugated operators $Q_{\tau}(\varepsilon x, D)=e^{\tau w} Q(\varepsilon x, D) e^{-\tau w}$ are uniformly subelliptic with loss of $(n-1) / n$ derivatives measured in $L^{p}, p=2 n /(n+2)$. This is fortunate, since, as we shall see, the proof of Theorem 1.1 requires subellipticity with loss of $\delta<1$ derivatives in $L^{p}$.

Before proving these Carleman inequalities let us first see why they imply Theorem 1.1. To do this let $Q(x, D)$ now be a second order elliptic operator which we only assume to be of the form

$$
Q(x, D)=\sum_{j, k=1}^{n} g^{j k}(x) D_{j} D_{k}
$$


with the $g^{j k}$ being $C^{\infty}$ and real. Also suppose that

$$
|Q(x, D) u| \leq|V u|+\left|V^{\prime} \cdot \nabla u\right|
$$

in a connected open set where $V \in L_{\mathrm{loc}}^{n / 2}, V^{\prime} \in L_{\mathrm{loc}}^{\infty}$ and $u$ satisfies $D^{\alpha} u \in L_{\mathrm{loc}}^{p}$, $0 \leq|\alpha| \leq 2$. Then, after possibly making an initial change of variables, one sees that Theorem 1.1 would follow if we could show that if $X_{0}$ is a neighborhood of 0 and

$$
\text { supp } u \cap\left\{x \in X_{0}: x_{1} \geq 0\right\} \subset\{0\},
$$

then $0 \notin \operatorname{supp} u$. Finally, by making a proper choice of geodesic cartesian coordinates we may assume that $Q(x, D)$ is as in (3.1) and that (3.4) and (3.5) still hold (see [5; 9, vol. III, p. 500]). Note that even though first order terms will arise from the change of coordinates, their contribution can be absorbed in (3.4).

Having made these reductions, we now are in a position to argue essentially as in [9, Chapter 17]. First set $u_{\varepsilon}(x)=u(\varepsilon x)$ where $\varepsilon$ is chosen small enough so that (3.2) and (3.3) hold for $X=\{x:|x|<\varepsilon\}$. Let $\eta \in C_{0}^{\infty}(X)$ be equal to one when $|x|<\varepsilon / 2$ and set $U=\eta u_{\varepsilon}$. Then if $V_{\varepsilon}(x)=V(\varepsilon x)$

$$
Q(\varepsilon x, D) U=\varepsilon^{2} \eta(Q u)(\varepsilon x)+\sum_{0<|\alpha| \leq 2} D^{\alpha} \eta Q^{(\alpha)}(\varepsilon x, D) u_{\varepsilon} / \alpha !
$$

which implies that

$$
|Q(\varepsilon x, D) U| \leq C_{0}\left(1+\left|V_{\varepsilon}\right|\right)|U|+C_{0}|\nabla U|, \quad|x| \leq \varepsilon / 2 .
$$

To use this set

$$
S_{\rho}=\left\{x \in X:-\rho \leq x_{1} \leq 0\right\}, \quad \rho>0 .
$$

Then, by (3.5), we can always choose $\rho$ to be small enough so that

$$
S_{\rho} \cap \operatorname{supp} u \subset\{|x|<\varepsilon / 2\},
$$

and so that if $C$ is as in (3.2)-(3.3) and $C_{0}$ is as in (3.6) then

$$
C C_{0}\left(\int_{S_{\rho}}\left(1+\left|V_{\varepsilon}\right|\right)^{n / 2} d x\right)^{2 / n}<1 / 2 \text {. }
$$

Next, notice that (3.2) and (3.3) imply

$$
\begin{array}{rl}
\| e^{\tau w} & U\left\|_{L^{p^{\prime}}\left(S_{\rho}\right)}+\tau^{1 / n}\right\| e^{\tau w} \nabla U \|_{L^{p}\left(S_{\rho}\right)} \\
& \leq C\left\|e^{\tau w} Q(\varepsilon x, D) U\right\|_{L^{p}(X)} \\
& \leq C\left\|e^{\tau w} Q(\varepsilon x, D) U\right\|_{L^{p}\left(S_{\rho}\right)}+C\left\|e^{\tau w} Q(\varepsilon x, D) U\right\|_{\left.L^{p(c} S_{\rho}\right)} .
\end{array}
$$

But, if we recall that $1 / p-1 / p^{\prime}=2 / n$, then we see that (3.6) and Hölder's inequality imply

$$
\begin{array}{rl}
C \| e^{\tau w} & Q(\varepsilon x, D) U \|_{L^{p}\left(S_{\rho}\right)} \\
& \leq C C_{0}\left\|\left(1+\left|V_{\varepsilon}\right|\right) \cdot e^{\tau w} U\right\|_{L^{p}\left(S_{\rho}\right)}+C C_{0}\left\|e^{\tau w} \nabla U\right\|_{L^{p}\left(S_{\rho}\right)} \\
& \leq \frac{1}{2}\left\|e^{\tau w} U\right\|_{L^{p^{\prime}}\left(S_{\rho}\right)}+C C_{0}\left\|e^{\tau w} \nabla U\right\|_{L^{p}\left(S_{\rho}\right)} .
\end{array}
$$


Thus, by (3.5), we can conclude that whenever $\tau$ is sufficiently large

$$
\left\|e^{\tau w} U\right\|_{L^{p^{\prime}}\left(S_{\rho}\right)}+\left\|e^{\tau w} \nabla U\right\|_{L^{p}\left(S_{\rho}\right)} \leq 2 C\left\|e^{\tau w} Q(\varepsilon x, D) U\right\|_{L^{p}\left(\left\{x \in X: x_{1}<-\rho\right\}\right)} .
$$

Finally, since $w^{\prime}(x)=1+x_{1}>0$ on $X$, this forces $U(x)=0$ when $x \in S_{\rho}$ and so $0 \notin \operatorname{supp} u$ which completes the proof.

Proof of Theorem 3.1. As we pointed out before, the proof of Theorem 3.1 is modelled after that of Theorem 2.1. So to prove (3.2) and (3.3) we first notice that if we set $v=e^{\tau w} u$ and

$$
Q_{\tau}(\varepsilon x, D)=e^{\tau w} Q(\varepsilon x, D) e^{-\tau w}
$$

then the desired inequalities would be a consequence of the following inequalities:

$$
\begin{gathered}
\|v\|_{p^{\prime}} \leq C\left\|Q_{\tau}(\varepsilon x, D) v\right\|_{p}, \\
\sum_{|\alpha| \leq 1} \tau^{(1+1 / n)-|\alpha|}\left\|D^{\alpha} v\right\|_{p} \leq C\left\|Q_{\tau}(\varepsilon x, D) v\right\|_{p} .
\end{gathered}
$$

To prove these we notice that

$$
Q_{\tau}(\varepsilon x, D)=Q(\varepsilon x, D)+2 \tau w^{\prime} \frac{\partial}{\partial x_{1}}-\left(\tau w^{\prime}\right)^{2}+\tau w^{\prime \prime}
$$

Consequently, since $w^{\prime}=1+x_{1} \approx 1$ on $X$, it follows that, if we let $A_{\tau}(\varepsilon x, D)$ be the differential operator whose adjoint equals

$$
A_{\tau}^{*}(\varepsilon x, D)=\left(\frac{1}{w^{\prime}(x)}\right)^{2} Q(\varepsilon x, D)-2 \tau \frac{1}{w^{\prime}(x)} \frac{\partial}{\partial x_{1}}-\tau^{2},
$$

then it suffices to prove the following variant of $\left(3.2^{\prime}\right)-\left(3.3^{\prime}\right)$ :

$$
\begin{aligned}
\|v\|_{p^{\prime}} & \leq C\left\|A_{\tau}(\varepsilon x, D) v\right\|_{p}, \\
\sum_{|\alpha| \leq 1} \tau^{(1+1 / n)-|\alpha|}\left\|D^{\alpha} v\right\|_{p} & \leq C\left\|A_{\tau}(\varepsilon x, D) v\right\|_{p}, \quad v \in C_{0}^{\infty}(X) .
\end{aligned}
$$

As before, we have made these reductions to simplify the notation later on.

As in $\S 2$, the desired inequalities will follow if we could construct a right parametrix, $T$, for $A_{\tau}^{*}(\varepsilon x, D)$ satisfying certain $L^{p}$ estimates. These are contained in the following lemma.

Lemma 3.3. For $\tau>0$ there are functions $K=K_{\tau}$ and $R=R_{\tau}$ so that

$$
A_{\tau}^{*}(\varepsilon x, D) K(x, y)=\delta_{0}(x-y)+R(x, y), \quad x, y \in X,
$$

where $\delta_{0}$ denotes the Dirac distribution. Furthermore, if we let $T=T_{\tau}$ be the operator with kernel $K(x, y)$ and $R$ be the operator with kernel $R(x, y)$, then, if $\varepsilon$ and $1 / \tau$ are sufficiently small, the adjoints of these operators satisfy the 
following uniform estimates whenever $f \in C_{0}^{\infty}(X)$ :

$$
\begin{gathered}
\left\|T^{*} f\right\|_{L^{p^{\prime}(X)}} \leq C\|f\|_{L^{p}(X)}, \\
\sum_{|\alpha| \leq 1} \tau^{(1+1 / n)-|\alpha|}\left\|D^{\alpha} T^{*} f\right\|_{L^{p}(X)} \leq C\|f\|_{L^{p}(X)}, \\
\tau^{1 / n}\left\|R^{*} f\right\|_{L^{q}(X)} \leq C\|f\|_{L^{q}(X)}, \quad q=p, p^{\prime}, \\
\tau^{-1+1 / n}\left\|\nabla R^{*} f\right\|_{L^{p}(X)} \leq C\|f\|_{L^{p}(X)} .
\end{gathered}
$$

Here $p$ and $p^{\prime}$ are as in Theorem 3.2.

Before proving Lemma 3.3 let us give the simple argument showing that (3.10)-(3.13) imply (3.8). First (3.9) implies the formula

$$
v(x)=T^{*}\left(A_{\tau}(\varepsilon \cdot, D) v\right)(x)-R^{*} v(x),
$$

and so Minkowski's inequality, (3.10) and (3.12) give that

$$
\begin{aligned}
\|v\|_{p^{\prime}} & \leq\left\|T^{*}\left(A_{\tau}(\varepsilon x, D) v\right)\right\|_{p^{\prime}}+\left\|R^{*} v\right\|_{p^{\prime}} \\
& \leq C\left\|A_{\tau}(\varepsilon x, D) v\right\|_{p}+C \tau^{-1 / n}\|v\|_{p^{\prime}},
\end{aligned}
$$

which implies the first inequality in (3.8) when $\tau$ is large enough. It is clear that the same type of argument using (3.11) and (3.12) give the inequality in (3.8) corresponding to $\alpha=0$. To prove the inequality corresponding to $|\alpha|=1$ we notice that (3.11), (3.13) give

$$
\begin{aligned}
\|\nabla v\|_{p} & \leq\left\|\nabla\left(T^{*}\left(A_{\tau}(\varepsilon x, D) v\right)\right)\right\|_{p}+\left\|\nabla R^{*} v\right\|_{p} \\
& \leq C \tau^{-1 / n}\left\|A_{\tau}(\varepsilon x, D) v\right\|_{p}+C \tau^{1-1 / n}\|v\|_{p}
\end{aligned}
$$

and since we have already argued that $\tau^{1+1 / n}\|v\|_{p} \leq C\left\|A_{\tau}(\varepsilon x, D) v\right\|_{p}$, the result follows. Thus, to finish the proof, we are left with demonstrating Lemma 3.3.

Proof of Lemma 3.3. The key step in the proof is to find a factorization of the symbol of $A_{\tau}^{*}(\varepsilon x, D)$ that will allow us to microlocally invert $A_{\tau}^{*}(\varepsilon x, D)$ near the set where $A_{\tau}^{*}(\varepsilon x, \xi)$ vanishes. With this in mind, we recall (3.7) and (3.1) and define a symbol that depends only on the last $(n-1) \xi$ variables, $\xi^{\prime}$, as follows

$$
a\left(x, \xi^{\prime}\right)=\sqrt{\sum_{j, k \geq 2} g^{j k}(x) \xi_{j} \xi_{k}} .
$$

Now, as in $\S 2$, we can factor $A_{\tau}^{*}(\varepsilon x, \xi)$ :

$$
A_{\tau}^{*}(\varepsilon x, \xi)=B_{\tau}(\varepsilon x, \xi) \cdot G_{\tau}(\varepsilon x, \xi),
$$

where, this time,

$$
B_{\tau}(\varepsilon x, \xi)=\frac{1}{w^{\prime}(x)} \xi_{1}+i\left(\frac{1}{w^{\prime}(x)} a\left(\varepsilon x, \xi^{\prime}\right)-\tau\right),
$$


and

$$
G_{\tau}(\varepsilon x, \xi)=\frac{1}{w^{\prime}(x)} \xi_{1}-i\left(\frac{1}{w^{\prime}(x)} a\left(\varepsilon x, \xi^{\prime}\right)+\tau\right) .
$$

For later reference, we note that the ellipticity of $Q(x, D)$ implies that the "good" factor $G_{\tau}(\varepsilon x, \xi)$ never vanishes. In fact $a\left(\varepsilon x, \xi^{\prime}\right) \approx\left|\xi^{\prime}\right|$ and so there is a constant $c>0$ so that

$$
\left|G_{\tau}(\varepsilon x, \xi)\right| \geq c(|\xi|+\tau), \quad(x, \xi) \in X \times \mathbb{R}^{n} .
$$

One can only obtain such control of the "bad" factor, $B_{\tau}(\varepsilon x, \xi)$, however, away from a lower dimensional set. In fact, if we let

$$
\Sigma_{\tau}=\left\{(x, \xi) \in X \times \mathbb{R}^{n}: \xi_{1}=0, a\left(\varepsilon x, \xi^{\prime}\right)=w^{\prime}(x) \tau\right\},
$$

then $B_{\tau}(\varepsilon x, \xi)$ vanishes on $\Sigma_{\tau}$. Nonetheless, $B_{\tau}(\varepsilon x, \xi)$, and hence $A_{\tau}^{*}(\varepsilon x, \xi)$, can be bounded below when $(x, \xi)$ is sufficiently far away.

For instance, if we assume, as we may, that

$$
Q(0, D)=-\Delta
$$

and define

$$
\Gamma_{\tau}=\left\{(x, \xi) \in X \times \mathbb{R}^{n}:\left|\xi^{\prime}\right| \in[\tau / 4,4 \tau],\left|\xi_{1}\right| \leq \tau / 4\right\},
$$

then it follows that, whenever $\varepsilon$ is smaller than some fixed constant, there must be a $c>0$ so that

$$
\left|A_{\tau}^{*}(\varepsilon x, \xi)\right| \geq c\left(|\xi|^{2}+\tau^{2}\right) \quad \text { when }(x, \xi) \in{ }^{c} \Gamma_{\tau} .
$$

As in $\S 2$, we can use (3.20) to microlocally invert $A_{\tau}^{*}(\varepsilon x, D)$ away from $\Gamma_{\tau}$. To do this we first fix $\beta \in C_{0}^{\infty}(\mathbb{R})$ satisfying $\beta(s)=1$ if $|s| \in[1 / 4,4]$ and $\beta=0$ near $s=0$. We then define $\beta_{0}=\beta_{0, \tau}$ by

$$
\beta_{0}(\xi)=1-\beta\left(\left|\xi^{\prime}\right| / \tau\right) \beta\left(1-\xi_{1} / \tau\right)
$$

and notice that

$$
\beta_{0}(\xi)=0 \text { on } \Gamma_{\tau} .
$$

Consequently, if we define

$$
K_{0}(x, y)=(2 \pi)^{-n} \int \beta_{0}(\xi) \frac{e^{i\langle x-y, \xi\rangle}}{A_{\tau}^{*}(\varepsilon y, \xi)} d \xi
$$

and recall (3.20), then we can conclude that standard microlocal arguments (cf. [9, Chapter 19]) give that

$$
A_{\tau}^{*}(\varepsilon x, D) K_{0}(x, y)=(2 \pi)^{-n} \int \beta_{0}(\xi) e^{i\langle x-y, \xi\rangle} d \xi+R_{0}(x, y),
$$

where $R_{0}$ belongs to a bounded subset of $S^{-1}$ that is independent of $\tau$ (see Definition 2.4). Thus, since the adjoint operator, $R_{0}^{*}$, also has this property it 
follows from standard results concerning the $L^{p}$ boundedness of pseudodifferential operators (see [19]) that when $f \in C_{0}^{\infty}(X)$

$$
\left\|\nabla R_{0}^{*} f\right\|_{L^{p}(X)} \leq C\|f\|_{L^{p}(X)},
$$

which is better than the bounds in (3.13) when $\tau$ is large. Furthermore, since one can also check that $\tau R_{0}$ belongs to a bounded subset of $S^{0}$, it follows that

$$
\tau\left\|R_{0}^{*} f\right\|_{L^{q}(X)} \leq C\|f\|_{L^{q}(X)}, \quad q=p, p^{\prime} .
$$

Next, let $T_{0}$ be the operator with kernel $K_{0}(x, y)$. Then since (3.20) and (3.22) imply that $\tau^{2} T_{0}$ belongs to a bounded subset of $S^{0}$, it must follow that

$$
\sum_{|\alpha| \leq 1} \tau^{2-|\alpha|}\left\|D^{\alpha} T_{0}^{*} f\right\|_{L^{p}(X)} \leq C\|f\|_{L^{p}(X)},
$$

which also is stronger than the corresponding inequality in Lemma 3.3. Finally, since $T_{0} \in S^{-2}$ and $1 / p-1 / p^{\prime}=2 / n$, it follows from the Sobolev imbedding theorem (see [19]) that

$$
\left\|T_{0}^{*} f\right\|_{L^{p^{\prime}(X)}} \leq C\|f\|_{L^{p}(X)},
$$

whenever, as above, $f \in C_{0}^{\infty}(X)$. Thus we have shown that our microlocal inverse corresponding to ${ }^{c} \Gamma_{\tau}$ satisfies the desired estimates.

To invert $A_{\tau}^{*}(\varepsilon x, D)$ on the set $\Gamma_{\tau}$ we first let $\beta_{1}=\beta_{1, \tau}$ be defined by

$$
\beta_{1}(\xi)=1-\beta_{0}(\xi),
$$

where $\beta_{0}$ is as in (3.21). As before, we would like to construct a Fourier integral operator, $T_{1}$, with kernel

$$
K_{1}(x, y)=(2 \pi)^{-n} \int \beta_{1}(\xi) \frac{e^{i \Phi(x, y, \xi)}}{A_{\tau}^{*}(\varepsilon y, \xi)} d \xi
$$

so that the analogues of (3.23) and (3.10)-(3.13) are satisfied. It is for this step that the factorization $(3.15)$ of the symbol $A_{\tau}^{*}(\varepsilon x, \xi)$ will be used.

Since the factor $G_{\tau}(\varepsilon x, \xi)$ is bounded below on $\Gamma_{\tau}$, and in fact satisfies (3.18), the previous discussions indicate that we should try to construct the phase function in (3.24) using the other factor in (3.15), $B_{\tau}(\varepsilon x, \xi)$. Ideally we would like $\Phi(x, y, \xi)$ to solve the complex eikonal equation

$$
B_{\tau}\left(\varepsilon x, \Phi_{x}\right)=B_{\tau}(\varepsilon y, \xi), \quad x, y \in X, \xi \in \operatorname{supp} \beta_{1} .
$$

However, since this is a nonlinear equation with only $C^{\infty}$ and not analytic coefficients, in general a solution does not exist. Nonetheless using the almost analytic machinery of Treves [20, Chapter 11], one can construct approximate solutions to (3.25) which will turn out to be sufficient for our purposes. In the following lemma we collect the properties of such a solution to (3.25). Since the construction of $\Phi$ involves only straightforward modifications of similar ones in Treves [20] the proof of Lemma 3.4 will only be sketched in an appendix. 
Lemma 3.4. As above, let $X=\left\{x \in \mathbb{R}^{n}:|x|<\varepsilon\right\}$. Then if $\varepsilon$ is small enough, there is a $C^{\infty}$ approximate solution $\Phi(x, y, \xi)$ to (3.25) which has nonnegative imaginary part, is homogeneous of degree one in $\xi$, and satisfies:

$$
\left|B_{\tau}\left(\varepsilon x, \Phi_{x}\right)-B_{\tau}(\varepsilon y, \xi)\right| \leq C_{N}(\operatorname{Im} \Phi(x, y, \xi))^{N}|\xi|^{1-N},
$$

for every $N$. Furthermore, $\Phi$ can be written as

$$
\Phi(x, y, \xi)=\phi\left(x^{\prime}, y, \xi^{\prime}\right)+\psi(x, y, \xi),
$$

where $\phi$ is real and satisfies

$$
\phi\left(x^{\prime}, y, \xi^{\prime}\right)=\left\langle x^{\prime}-y^{\prime}, \xi^{\prime}\right\rangle+O\left(\left|x^{\prime}-y^{\prime}\right|^{2}\left|\xi^{\prime}\right|\right),
$$

while $\psi$ satisfies

$$
\psi(x, y, \xi)=\left(x_{1}-y_{1}\right) \xi_{1}+O\left(\left|x_{1}-y_{1}\right|^{2}\left|\xi^{\prime}\right|\right)
$$

and

$$
\operatorname{Im} \psi(x, y, \xi) \geq c\left(x_{1}-y_{1}\right)^{2}\left|\xi^{\prime}\right|
$$

(when $\beta_{1}(\xi) \neq 0$ ) for some positive constant $c$.

Remark 3.5. Notice that, since $a\left(x, \xi^{\prime}\right)$ defines an analytic function of $\xi^{\prime}$ in some conic region of $\mathbb{C}^{n-1}$ containing $\mathbb{R}^{n-1}, B_{\tau}\left(\varepsilon x, \Phi_{x}\right)$ and $G_{\tau}\left(\varepsilon x, \Phi_{x}\right)$ are both well defined when $x, y \in X$ and $\varepsilon$ is small enough.

To apply Lemma 3.4, we note that if $\Phi$ is the phase function there then (3.7) implies that

$$
A_{\tau}^{*}(\varepsilon x, D) e^{i \Phi(x, y, \xi)}=e^{i \Phi} A_{\tau}^{*}\left(\varepsilon x, \Phi_{x}\right)+e^{i \Phi}\left(\frac{1}{w^{\prime}(x)}\right)^{2} Q(\varepsilon x, D) \Phi .
$$

Next, if we set

$$
\begin{aligned}
r(x, y, \xi)= & G_{\tau}(\varepsilon y, \xi)-G_{\tau}(\varepsilon x, \xi) \\
= & \left(\frac{1}{w^{\prime}(y)} \xi_{1}-i \frac{1}{w^{\prime}(y)} a\left(\varepsilon y, \xi^{\prime}\right)\right) \\
& -\left(\frac{1}{w^{\prime}(x)}-i \frac{1}{w^{\prime}(x)} a\left(\varepsilon x, \xi^{\prime}\right)\right)
\end{aligned}
$$

then it follows from (3.15), (3.16) and (3.30) that

$$
e^{i \Phi} A_{\tau}^{*}\left(\varepsilon x, \Phi_{x}\right)=e^{i \Phi} A_{\tau}^{*}(\varepsilon y, \xi)+e^{i \Phi} B_{\tau}(\varepsilon y, \xi) r(x, y, \xi)+O\left(\tau^{-N}\right)
$$

for every $N$ when $\beta_{1}(\xi) \neq 0$. Consequently, (3.31)-(3.33) imply that if $K_{1}$ is as in (3.24) then

$$
\begin{aligned}
(2 \pi)^{n} & A_{\tau}^{*}(\varepsilon x, D) K_{1}(x, y) \\
= & \int \beta_{1}(\xi) e^{i \Phi} d \xi+\int \beta_{1}(\xi) \frac{r(x, y, \xi)}{G_{\tau}(\varepsilon y, \xi)} e^{i \Phi} d \xi \\
& +\frac{1}{\left(w^{\prime}(x)\right)^{2}} \int \beta_{1}(\xi) \frac{Q(\varepsilon x, D) \Phi}{A_{\tau}^{*}(\varepsilon y, \xi)} e^{i \Phi} d \xi+O\left(\tau^{-N}\right)
\end{aligned}
$$


Next, since the phase function $\Phi$ satisfies

$$
\nabla_{\xi} \Phi(x, y, \xi) \neq 0 \quad \text { unless } x=y
$$

it follows from the theory of Fourier integrals (see [6, pp. 201-202; 20,§17.6]) that

$$
\int \beta_{1}(\xi) e^{i \Phi(x, y, \xi)} d \xi-\int \beta_{1}(\xi) e^{i\langle x-y, \xi\rangle} d \xi
$$

belongs to a bounded subset of $S^{-1}$, and, further, on account of the support properties of $\beta_{1}(\xi), \tau$ times this difference belongs to a bounded subset of $S^{0}$. Similarly, since $r(x, y, \xi) \in S^{1}$ and

$$
r(x, y, \xi)=0 \text { when } x=y,
$$

it also follows from (3.35) that the second summand in (3.34) also has these properties, and the last summand in (3.34) trivially has these properties. Putting all this together, we see that $(3.34)$ can be rewritten as

$$
A_{\tau}^{*}(\varepsilon x, D) K_{1}(x, y)=(2 \pi)^{-n} \int \beta_{1}(\xi) e^{i\langle x-y, \xi\rangle} d \xi+R_{1,0}(x, y)+R_{1,1}(x, y),
$$

where

$$
R_{1,1}(x, y)=\frac{(2 \pi)^{-n}}{\left(w^{\prime}(x)\right)^{2}} \int \beta_{1}(\xi) \frac{Q(\varepsilon x, D) \Phi}{A_{\tau}^{*}(\varepsilon y, \xi)} e^{i \Phi} d \xi,
$$

while $R_{1,0}$ belongs to a bounded subset of $S^{-1}$ and $\tau R_{1,0}$ belongs to a bounded subset of $S^{0}$.

On account of this formula, we see that if we let $K(x, y)=K_{0}(x, y)+$ $K_{1}(x, y)$ and $R(x, y)=R_{0}(x, y)+R_{1}(x, y)$, where $R_{1}=R_{1,0}+R_{1,1}$, then (3.9) must hold. Thus, since $R_{1,0}$ satisfies the desired estimates, we see from Minkowski's inequality that, in order to finish the proof of Lemma 3.3, it suffices to show that for $f \in C_{0}^{\infty}(X)$ one has

$$
\begin{gathered}
\left\|T_{1}^{*} f\right\|_{L^{\prime}(X)} \leq C\|f\|_{L^{p}(X)}, \\
\sum_{|\alpha| \leq 1} \tau^{(1+1 / n)-|\alpha|}\left\|D^{\alpha} T_{1}^{*} f\right\|_{L^{p}(X)} \leq C\|f\|_{L^{p}(X)}, \\
\tau^{1 / n}\left\|R_{1,1}^{*} f\right\|_{L^{p}(X)} \leq C\|f\|_{L^{q}(X)}, \quad q=p, p^{\prime}, \\
\tau^{-1+1 / n}\left\|\nabla R_{1,1}^{*} f\right\|_{L^{p}(X)} \leq C\|f\|_{L^{p}(X)} .
\end{gathered}
$$

(Here $T_{1}$ denotes the operator with kernel $K_{1}$.)

In $\S 2$ we were able to prove the desired $L^{2}$ estimates for $T_{1}$ using Plancherel's theorem for $\mathbb{R}^{n-1}$ and a partial integration argument. To prove the desired $L^{p}$ estimates we shall also use a partial integration argument; however, here we shall need to invoke the following oscillatory integral theorem of Carleson and Sjölin [3] and Stein [18] which will serve as a substitute for the Plancherel theorem. 
Lemma 3.6. Let $a\left(x^{\prime}, y^{\prime}\right)$ be a $C^{\infty}$ function supported in the set $\left\{\left(x^{\prime}, y^{\prime}\right) \in\right.$ $\left.\mathbb{R}^{n-1} \times \mathbb{R}^{n-1}:\left|x^{\prime}\right|<1 / 4,1 / 2<\left|y^{\prime}\right|<2\right\}$. Then if for $\lambda>0$ we set

$$
R_{\lambda} f\left(x^{\prime}\right)=\int_{\mathbb{R}^{n-1}} e^{i \lambda \varphi\left(x^{\prime}, y^{\prime}\right)} a\left(x^{\prime}, y^{\prime}\right) f\left(y^{\prime}\right) d y^{\prime},
$$

it follows that for all (real) functions $\varphi$ belonging to some fixed neighborhood $\mathscr{N}$ of the function $\varphi_{0}\left(x^{\prime}, y^{\prime}\right)=\left|x^{\prime}-y^{\prime}\right|$ in the $C^{\infty}$ topology

$$
\begin{gathered}
\left\|R_{\lambda} f\right\|_{p^{\prime}} \leq C \lambda^{-(n-2) / p^{\prime}}\|f\|_{p}, \\
\left\|R_{\lambda} f\right\|_{q} \leq C \lambda^{-(n-1) / p^{\prime}}\|f\|_{q}, \quad q=p, p^{\prime},
\end{gathered}
$$

if $p$ and $p^{\prime}$ are as above. The constant $C$ depends only on the size of finitely many derivatives of the function $a$.

To apply Lemma 3.6, we shall need to know more about the kernel $K_{1}(x, y)$. In the following lemma we collect the necessary facts.

Lemma 3.7. The kernel $K_{1}(x, y)$ can be written as

$$
K_{1}(x, y)=\sum_{j=0,1} a_{j}(x, y) \frac{\tau^{n-2} e^{i \tau \varphi_{j}\left(x^{\prime}, y\right)}}{\left|\tau\left(x^{\prime}-y^{\prime}\right)\right|^{(n-2) / 2}|\tau(x-y)|},
$$

where, for every fixed $N$, the factors $a_{j}$ satisfy

$$
\left|D^{\alpha} a_{j}(x, y)\right| \leq C_{\alpha}\left(1+\tau\left(x_{1}-y_{1}\right)^{2}\right)^{-N}\left|x^{\prime}-y^{\prime}\right|^{-|\alpha|},
$$

and, furthermore, the phase functions $\varphi_{j}$ are real and have the property that when $\varepsilon$ is small enough, $0<\rho \leq \varepsilon$ and $y_{1} \in[-\varepsilon, \varepsilon]$ is fixed, the dilated functions

$$
\left(x^{\prime}, y^{\prime}\right) \rightarrow(-1)^{j} \rho^{-1} \varphi_{j}\left(\rho x^{\prime}, y_{1}, \rho y^{\prime}\right) \in \mathcal{N},
$$

where $\mathscr{N}$ is the set in Lemma 3.6. Also, one has the uniform estimates

$$
\left|K_{1}(x, y)\right| \leq C \tau^{n-2}\left(1+\tau\left|x_{1}-y_{1}\right|\right)^{-1}
$$

Remark 3.8. The phase functions satisfy (3.42) since we are assuming that $Q(0, D)=-\Delta$. Also, recall that the kernel of the ball multiplier operators in $\mathbb{R}^{n-1}$,

behave asymptotically like

$$
\int_{\left|\xi^{\prime}\right| \leq \tau} e^{i\left\langle x^{\prime}-y^{\prime}, \xi^{\prime}\right\rangle} d \xi^{\prime}
$$

$$
\frac{\tau^{n-1} e^{i \tau\left|x^{\prime}-y^{\prime}\right|}}{\left|\tau\left(x^{\prime}-y^{\prime}\right)\right|^{n / 2}}
$$

(plus another term with $\left|x^{\prime}-y^{\prime}\right|$ being replaced by $-\left|x^{\prime}-y^{\prime}\right|$ in the exponential). Thus, Lemma 3.7 tells us that the kernel $K_{1}(x, y)$ is basically the product of $\tau^{-3 / 2}$ times the product of the kernel of an approximation to the identity of width $\tau^{-1 / 2}$ in the first variable and a ball multiplier operator kernel in the other 
variables. On account of this, the reason that the bounds in the $L^{p}$ Carleman inequality (3.3) are worse than the corresponding $L^{2}$ Carleman inequality (2.2) is related to the fact that the above ball multiplier operators map $L^{p}\left(\mathbb{R}^{n-1}\right)$ functions with fixed compact support to $L_{\text {loc }}^{p}$ with norm comparable to $\tau^{1 / 2-1 / n}$ when $p$ is as above. (For results of a similar nature see $[15,17]$.)

Proof of Lemma 3.7. For simplicity, let us first assume that we are in the ideal case where $Q(x, D)=-\Delta$, and in this case, let us assume that $x=0$. Then, by a change of scale, the kernel would equal

where $\Phi$ is as in (2.24).

$$
\tau^{n-2} \int_{\mathbb{R}^{n}} \beta_{1}(\tau \xi) \frac{e^{i \tau \Phi(x, y, \xi)}}{|\xi|^{2}-2 i \xi_{1}-1} d \xi
$$

To compute this oscillatory integral, we express $\xi^{\prime}$ in polar coordinates $\xi^{\prime}=$ $r \omega^{\prime}$, with $\omega^{\prime} \in \mathbf{S}^{n-2}$ and recall that

$$
\int_{\mathbf{S}^{n-2}} \beta_{1}(\tau \xi) e^{i \tau r\left\langle x^{\prime}-y^{\prime}, \omega^{\prime}\right\rangle} d \omega^{\prime}=\sum_{ \pm} a_{ \pm} \frac{e^{i \pm \tau r\left|x^{\prime}-y^{\prime}\right|}}{\left|\tau\left(x^{\prime}-y^{\prime}\right)\right|^{(n-2) / 2}}
$$

for functions $a_{ \pm}$satisfying $\left|D^{\alpha} a_{ \pm}\right| \leq C_{\alpha}\left|x^{\prime}-y^{\prime}\right|^{-|\alpha|}$. Next, recall that $\beta_{1}(\tau \xi)=$ 0 when $\left|\xi^{\prime}\right| \notin[1 / 4,4]$. This is fortunate since it is easy to check that when $b\left(\xi_{1}, r\right)$ in $C_{0}^{\infty}(\mathbb{R} \times \mathbb{R})$ has $r$ support in $[1 / 4,4]$

$$
\iint b\left(\xi_{1}, r\right) \frac{e^{i \tau r\left|x^{\prime}-y^{\prime}\right|} e^{i \tau\left[\xi_{1}\left(x_{1}-y_{1}\right)+\left(x_{1}-y_{1}\right)^{2}\left(i r+\xi_{1}\right) / 2\right]}}{r^{2}+\xi_{1}^{2}-2 i \xi_{1}-1} d r d \xi_{1}=\frac{a(x, y)}{|\tau(x-y)|}
$$

for a function $a$ satisfying (3.41). Finally, if one combines the last two identities, then one sees results in Lemma 3.7 hold for this special case.

The proof for the general case follows from a similar argument after one takes note of the fact that if $\phi$ is as in (3.28) and $h$ belongs to a bounded subset of $C^{\infty}\left(\mathbf{S}^{n-2}\right)$

$$
\int_{\mathbf{S}^{n-2}} e^{i \tau \phi\left(x^{\prime}, y, \omega^{\prime}\right)} h\left(\omega^{\prime}\right) d \omega^{\prime}=\sum_{j=0,1} a_{j} \frac{e^{i \tau \varphi_{j}\left(x^{\prime}, y\right)}}{\left|\tau\left(x^{\prime}-y^{\prime}\right)\right|^{(n-2) / 2}},
$$

where the $\varphi_{j}$ are as in Lemma 3.7 and $\left|D^{\alpha} a_{j}\right| \leq C_{\alpha}\left|x^{\prime}-y^{\prime}\right|^{-|\alpha|}$. This just follows from standard stationary phase results, such as [9, Theorem 7.7.6], and the fact that $\phi$ satisfies (3.28), and hence, for $x^{\prime}$ close to $y^{\prime}$, is a small perturbation of $\left\langle x^{\prime}-y^{\prime}, \xi^{\prime}\right\rangle$. This is half of the argument. The other estimate which involves $\xi_{1}$ and a radial integration goes exactly as before. One gets the rapid decay (3.41) since we have lower bounds (3.30) on the imaginary part of $\Phi$.

End of proof of Lemma 3.3. First let $\eta \in C_{0}^{\infty}(\mathbb{R})$ be supported in $[1 / 4,4]$ and have the property that $\sum_{-\infty}^{\infty} \eta\left(2^{\nu} s\right)=1, s>0$, and set $\eta_{0}(s)=1-$ $\sum_{-\infty}^{0} \eta\left(2^{\nu} s\right)$. Then we define kernels $K_{1, \nu}, \nu=0,1,2, \ldots$, as follows

$$
K_{1, \nu}(x, y)= \begin{cases}\eta\left(\tau 2^{-\nu}\left|x^{\prime}-y^{\prime}\right|\right) K_{1}(x, y), & \nu>0, \\ \eta_{0}\left(\tau\left|x^{\prime}-y^{\prime}\right|\right) K_{1}(x, y), & \nu=0 .\end{cases}
$$


We claim that, if $T_{1, \nu}$ are the operators associated to these kernels, then Lemmas 3.6 and 3.7 imply that for functions supported in $X$ one has the following estimates:

$$
\begin{gathered}
\left\|T_{1, \nu}^{*} f\right\|_{L^{p^{\prime}(X)}} \leq C 2^{-2 \nu / n}\|f\|_{L^{p}(X)}, \\
\left\|T_{1, \nu}^{*} f\right\|_{L^{p}(X)} \leq C\left(\tau 2^{-\nu}\right)^{-1 / p^{\prime}} \tau^{-(1+1 / n)}\|f\|_{L^{p}(X)} .
\end{gathered}
$$

By summing a geometric series one sees that these estimates imply (3.10.1) as well as (3.11.1) for the special case $\alpha=0$.

Let us first see why (3.44) holds. One can check that (3.43) implies that the $L^{r}$ norm of $K_{1,0}^{*}$ with respect to either of the variables is $O\left(\tau^{n-2} \tau^{-n / r}\right)$. But, if we let $r=n /(n-2)$, it then follows from Young's inequality, and the fact that $1 / p-1 / p^{\prime}=2 / n$, that

$$
\left\|T_{1,0}^{*} f\right\|_{p^{\prime}} \leq C \tau^{n-2} \tau^{-n / r}\|f\|_{p}=C\|f\|_{p}
$$

as desired.

To prove the result for $\nu>0$, set $X^{\prime}=\left\{\left|x^{\prime}\right|<\varepsilon\right\}$ and let $K_{1, \nu}^{*}$ be the kernel of the operator $T_{1, \nu}^{*}$. Then, if we fix $x_{1}$ and $y_{1}$, it follows that the $L^{p}\left(X^{\prime}\right) \rightarrow L^{p^{\prime}}\left(X^{\prime}\right)$ norm of the operator

$$
\left(T_{1, \nu}^{\prime} g\right)\left(x^{\prime}\right)=\int_{X^{\prime}} K_{1, \nu}^{*}(x, y) g\left(y^{\prime}\right) d y^{\prime}
$$

equals $\left(2^{\nu} / \tau\right)^{(n-1)\left(1-1 / p+1 / p^{\prime}\right)}$ times the norm of the dilated operator

$$
\left(\widetilde{T}_{1, \nu}^{\prime} g\right)\left(x^{\prime}\right)=\int K_{1, \nu}^{*}\left(x_{1}, \rho x^{\prime}, y_{1}, \rho y^{\prime}\right) g\left(y^{\prime}\right) d y^{\prime},
$$

where we have set $\rho=2^{\nu} / \tau$. However, by Lemma 3.7, the kernel in the last integral equals the complex conjugate of

$$
\tau^{n-2} \eta\left(\left|x^{\prime}-y^{\prime}\right|\right) \sum a_{j}\left(y_{1}, \rho y^{\prime}, x_{1}, \rho x^{\prime}\right) \frac{e^{i(\tau \rho) \cdot \rho^{-1} \varphi_{j}\left(\rho y^{\prime}, x_{1}, \rho x^{\prime}\right)}}{\left|\tau \rho\left(x^{\prime}-y^{\prime}\right)\right|^{(n-2) / 2}\left|\tau\left(x_{1}, \rho x^{\prime}, y_{1}, \rho y^{\prime}\right)\right|},
$$

and, consequently, one can use Lemma 3.6 to see that

$$
\left\|\widetilde{T}_{1, \nu}^{\prime} g\right\|_{p^{\prime}} \leq C(\tau \rho)^{-(n-2) / p^{\prime}} \cdot \tau^{n-2}(\tau \rho)^{-(n-2) / 2} \tau^{-1}\left(\left(x_{1}-y_{1}\right)^{2}+\rho^{2}\right)^{-1 / 2}\|g\|_{p},
$$

when $\rho$ is as in (3.42).

A little arithmetic, though, will now show that this implies the following estimate for $T_{1, \nu}^{\prime}$ :

$$
\begin{aligned}
& \left\|\int_{X^{\prime}} K_{1, \nu}^{*}(x, y) g\left(y^{\prime}\right) d y^{\prime}\right\|_{L^{p^{\prime}}\left(X^{\prime}\right)} \\
& \quad \leq C \tau^{-2 / n}\left(\left(x_{1}-y_{1}\right)^{2}+\left(2^{\nu} / \tau\right)^{2}\right)^{-1 / 2}\|g\|_{L^{p}\left(X^{\prime}\right)}
\end{aligned}
$$


whenever $g$ is supported in $X^{\prime}$. To use this notice that, if $r=n /(n-2)$, as above, then

$$
\left(\int_{-\infty}^{\infty}\left(\left(x_{1}-y_{1}\right)^{2}+\left(2^{\nu} / \tau\right)^{2}\right)^{-r / 2} d x_{1}\right)^{1 / r}=C\left(\tau / 2^{\nu}\right)^{2 / n}
$$

and so the desired estimate (3.44) follows from (3.46) and Young's inequality. (See $\S 2$ for a similar argument.)

The other inequality, (3.45), follows from a similar argument. In fact, as above, the case $\nu=0$ is trivial, while for $\nu>0$ one sees that Lemmas 3.6 and 3.7 along a change of scale argument give that

$$
\begin{aligned}
& \left\|\int_{X^{\prime}} K_{1, \nu}^{*}(x, y) g\left(y^{\prime}\right) d y^{\prime}\right\|_{L^{p}\left(X^{\prime}\right)} \\
& \quad \leq C \tau^{-1 / 2-1 / n}\left(\tau 2^{-\nu}\right)^{-1 / p^{\prime}}\left(1+\tau\left|x_{1}-y_{1}\right|^{2}\right)^{-2}\|g\|_{L^{p}\left(X^{\prime}\right)},
\end{aligned}
$$

which implies (3.45) since

$$
\int_{-\infty}^{\infty}\left(1+\tau\left|x_{1}-y_{1}\right|^{2}\right)^{-2} d x_{1}=C \tau^{-1 / 2}
$$

It is clear that these arguments will also prove the remaining estimates in (3.11.1)-(3.13.1) since $\nabla T_{1}^{*} \approx \tau T_{1}^{*}$ and $D^{\alpha} R_{1,1}^{*} \approx \tau^{1+|\alpha|} T_{1}^{*}$. This completes the proof.

\section{APPENDIX: THE PHASE FUNCTION CONSTRUCTION}

In this section we shall outline the construction of the phase function $\Phi(x, y, \xi)$ in Lemma 3.4 and sketch the proof of (3.26)-(3.30). The arguments are essentially in Treves [20, Chapter 11] to which we refer the reader for more details.

First we recall that $\Phi$ is to be an approximate solution to the complex eikonal equation

$$
B_{\tau}\left(\varepsilon x, \Phi_{x}\right)=B_{\tau}(\varepsilon y, \xi)
$$

which can be rewritten as

$\frac{1}{w^{\prime}(x)} \Phi_{x_{1}}(x, y, \xi)+\frac{i}{w^{\prime}(x)} a\left(\varepsilon x, \Phi_{x^{\prime}}(x, y, \xi)\right)=\frac{1}{w^{\prime}(y)} \xi_{1}+\frac{i}{w^{\prime}(y)} a\left(\varepsilon y, \xi^{\prime}\right)$.

As we noted before, this equation cannot in general be solved. However, the first step in finding an approximate solution is to solve the real eikonal equation

$$
a\left(\varepsilon y_{1}, \varepsilon x, \phi_{x^{\prime}}\left(x^{\prime}, y, \xi^{\prime}\right)\right)=a\left(\varepsilon y, \xi^{\prime}\right)
$$

obtained by "freezing" $x_{1}=y_{1}$. This equation can always be solved in a neighborhood of the diagonal $x^{\prime}=y^{\prime}$, and, in fact there is a unique real solution which vanishes when $\left\langle x^{\prime}-y^{\prime}, \xi^{\prime}\right\rangle=0$ and satisfies (3.28) (see e.g. [20, Chapter 6]). 
Next, if $\Phi$ is to be as in (3.27), then we are now led to study the equation

$$
\begin{aligned}
& \frac{1}{w^{\prime}(x)} \psi_{x_{1}}(x, y, \xi)+\frac{i}{w^{\prime}(x)} a\left(\varepsilon x, \phi_{x^{\prime}}\left(x^{\prime}, y, \xi^{\prime}\right)+\psi_{x^{\prime}}(x, y, \xi)\right) \\
& \quad=\frac{1}{w^{\prime}(y)} \xi_{1}+\frac{i}{w^{\prime}(y)} a\left(\varepsilon y, \xi^{\prime}\right)
\end{aligned}
$$

for an unknown function $\psi(x, y, \xi)$ which is to vanish when $x_{1}=y_{1}$. If we recall Remark 3.5, then it follows from [20, pp. 572-575] that, by choosing an almost analytic extension of $a\left(\varepsilon x, \xi^{\prime}\right)$ with respect to the $x$ variable, one can find an approximate solution having the property that, if $\varepsilon$ is small enough and $x, y \in X$, then the difference between the right-hand and left-hand side of the equation is $O\left(|\operatorname{Im} \psi|^{N}|\xi|^{1-N}\right)$ for every $N$ which implies (3.26). Furthermore, since one sees that $\psi$ is of the form (cf. (2.24)) $\psi(x, y, \xi)=\left(x_{1}-y_{1}\right) \xi_{1}+\frac{\left(x_{1}-y_{1}\right)^{2}}{2\left(1+y_{1}\right)} \xi_{1}+i \frac{\left(x_{1}-y_{1}\right)^{2}}{2\left(1+y_{1}\right)} a\left(\varepsilon y, \xi^{\prime}\right)+O\left(\varepsilon\left(x_{1}-y_{1}\right)^{2}\left|\xi^{\prime}\right|\right)$, the last two properties, (3.29) and (3.30), follow when $\varepsilon$ is small enough.

\section{REFERENCES}

1. S. Alinhac and M.S. Baouendi, Uniqueness for the characteristic Cauchy problem and strong unique continuation for higher order partial differential inequalities, Amer. J. Math. 102 (1980), 179-217.

2. M. Beals, C. Fefferman, and R. Grossman, Strictly pseudoconvex domains in $\mathbf{C}^{n}$, Bull. Amer. Math. Soc. (N.S.) 8 (1983), 125-322.

3. L. Carleson and P. Sjölin, Oscillatory integrals and a multiplier problem for the disc, Studia Math. 44 (1971), 287-299.

4. C. Fefferman, The multiplier problem for the ball, Ann. of Math. 94 (1971), 330-336.

5. H. Guggenheimer, Differential geometry, Dover, New York, 1977, pp. 329-335.

6. L. Hörmander, The spectral function of an elliptic operator, Acta Math. 88 (1968), 193-218.

7. __ Oscillatory integrals and multipliers on $F L^{p}$, Ark. Math. 11 (1971), 1-11.

8. __ Uniqueness theorems for second order elliptic differential equations, Comm. Partial Differential Equations 8 (1983), 21-64.

9. _ The analysis of linear partial differential operators, Vols. I-IV, Springer-Verlag, New York and Berlin, 1983, 1985.

10. D. Jerison, Carleman inequalities for the Dirac and Laplace operators and unique continuation, Adv. Math. 63 (1986), 118-134.

11. D. Jerison and C.E. Kenig, Unique continuation and absence of positive eigenvalues for Schrödinger operators, Ann. of Math. 121 (1985), 463-494.

12. C.E. Kenig, A. Ruiz, and C.D. Sogge, Uniform Sobolev inequalities and unique continuation for second order constant coefficient differential operators, Duke Math. J. 55 (1987), 329-347.

13. L. Nirenberg, Uniqueness in Cauchy problems for differential equations with constant leading coefficients, Comm. Pure Appl. Math. 10 (1957), 89-106.

14. E. Sawyer, Unique continuation for Schrödinger operators in dimensions three or less, Ann. Inst. Fourier (Grenoble) 33 (1984), 189-200.

15. C.D. Sogge, Oscillatory integrals and spherical harmonics, Duke Math. J. 53 (1986), 43-65.

16. _ Concerning the $L^{p}$ norm of spectral clusters for second order elliptic operators on compact manifolds, J. Funct. Anal. 77 (1988), 123-134.

17. $\ldots$, On the convergence of Riesz means on compact manifolds, Ann. of Math. 126 (1987), 439-447.

18. E. M. Stein, Oscillatory integrals in Fourier analysis, Beijing Lectures in Harmonic Analysis, Princeton Univ. Press, Princeton, NJ. 
19. M. Taylor, Pseudodifferential operators, Princeton Univ. Press, Princeton, NJ, 1981.

20. F. Treves, Introduction to pseudodifferential and Fourier integral operators, Vol. II, Plenum, New York, 1982.

21. __ Solution of Cauchy problems modulo flat problems, Comm. Partial Differential Equations 1 (1976), 45-72.

Department of Mathematics, University of Chicago, Chicago, Illinois 60637

Current address: Department of Mathematics, University of California, Los Angeles, California 90024 